\title{
Generation of hircine-induced pluripotent stem cells by somatic cell reprogramming
}

\author{
Cell Research (2011) 21:849-853. doi:10.1038/cr.2011.37; published online 15 March 2011
}

\section{Dear Editor,}

The goat has long been an important domesticated animal. Gene modification of goats to improve their production traits, ability to resist disease, and ability to produce valuable and high-quality proteins has been widely applied in the fields of agriculture and biopharming [1]. However, traditional transgenic techniques result in poorly controlled expression of exogenous genes and extremely low efficiency, greatly limiting the applications of genetically modified goats. Embryonic stem cells (ESCs) have been proved to be essential for gene targeting [2]. Gene targeting techniques based on ESCs, which have been well established in the mouse, allows precise and effective gene knockins and knockouts. However, it has been difficult to isolate and maintain ESCs from the embryos of goats and other ungulates due to little knowledge of the characteristics and culture conditions of ESCs from these species. Therefore, although many efforts have been made in the past few decades to derive goat ES cells, no authentic goat ESC line has been reported yet. Pluripotency could be obtained by reprogramming of somatic cells with defined factors [3]. More importantly, induced pluripotent stem (iPS) cells have been demonstrated as an efficient vehicle for precise genetic engineering in vivo [4], which means that iPS cells could be used as a substitute for ESCs to generate transgenic animals. Rat iPS cells were the first iPS cell lines generated before the report of rat ES cell lines. This is the proof-of-principle study to demonstrate that the iPS cell technique could be applied to species in which ES cell lines are difficult to generate [5]. Here, we report reprogramming of goat somatic cells by eight factors.

We have generated some goat iPS cell lines from goat primary ear fibroblasts (gPEFs) by constitutive expression of defined factors with lentiviral vectors carrying $\operatorname{OSCK}(O c t 3 / 4$, Sox2, c-Myc and Klf4). These cell lines exhibited some ES cell characteristics, including alkaline phosphatase (AP) activity, ES cell marker expression, and ability to form embryoid bodies (EB), including three germ layers. However, these cells failed to differ- entiate into three germ layers in teratoma, probably due to the constitutive expression of exogenous genes (data not shown). To solve this problem, we devised a reprogramming strategy in which transcription factors were expressed from doxycycline (DOX)-inducible lentiviral vectors [6]. Using inducible lentiviruses, the expression levels of the four factors were controlled by the addition of DOX to the culture medium.

Using this inducible expression strategy, we failed to generate goat iPS cells with viruses encoding cDNAs of Oct4, Sox2, cMyc, and Klf4 [3]. Viruses encoding Nanog and Lin 28 genes were then added to the infection cocktail without success [7]. The SV40 large T antigen and the catalytic subunit of human telomerase, hTERT, have been reported to significantly improve the efficiency of iPS cell generation [8]. Therefore, the genes for SV40 large $\mathrm{T}$ antigen and hTert were subsequently added to generate goat iPS cells. At 7 days after infection, colony-like cells appeared in the dishes. Since adding SV40 large T antigen and hTERT obviously increased the proliferation of cells, the transduced cells quickly formed very large colonies and became confluent. We then trypsinized the colonies into single cells and passaged them onto new dishes to release the well-reprogrammed cells. AP-positive cells with a compact colony morphology were observed at day 26 post infection (Figure 1A). The efficiencies of generating iPS cells were significantly different, when SV40 large T antigen was added individually or in combination with hTERT, which was confirmed by three independent experiments (Figure 1B). Unfortunately, we failed to obtain giPS cell lines in the absence of hTERT gene infection, as the cells lost pluripotency soon after the colonies were picked and passaged onto new feeder cells. Interestingly, we found that colony-like cells could not even be generated without SV40 large T antigen. We also could not generate competent iPS cells even with the SV40 large $T$ antigen in the absence of any of the other six proteins (Figure 1B).

giPSCs resemble mouse ES cells with a round and compact colony morphology. giPSCs show a nucleus/ cytoplasm ratio similar to ES cells. The doubling time of 
A

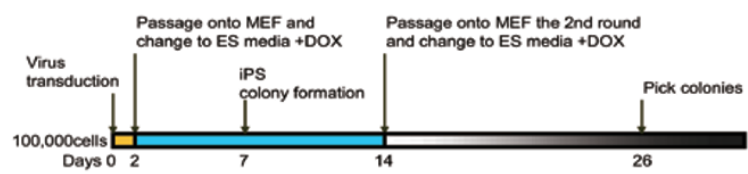

B

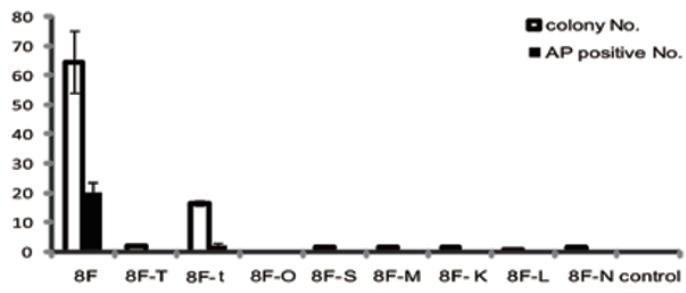

D

\section{E}
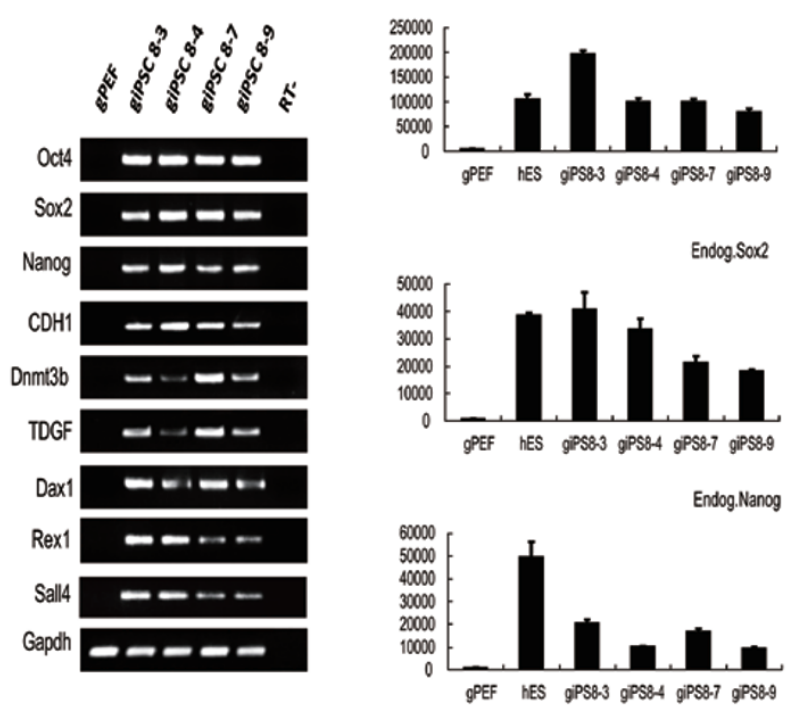

H
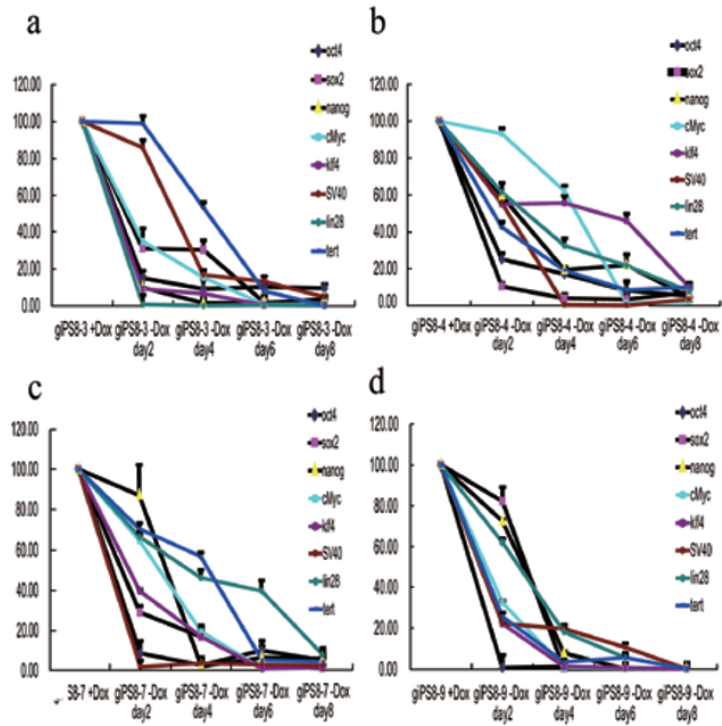

G

C
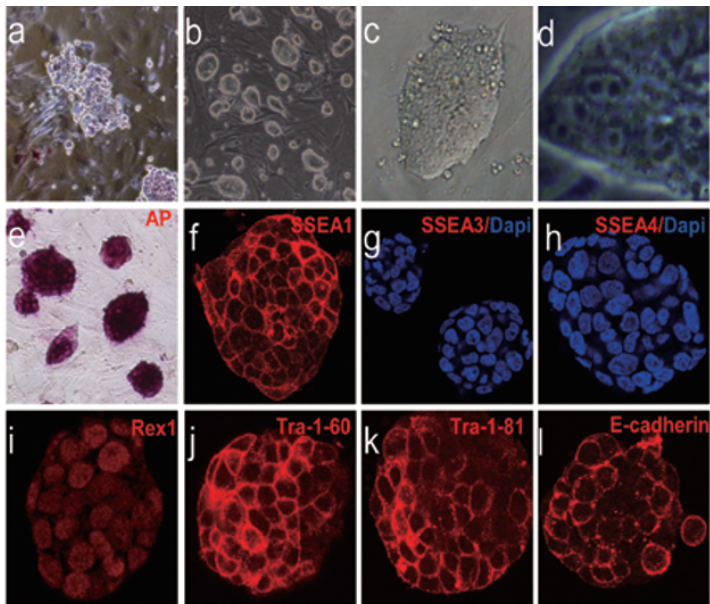

F
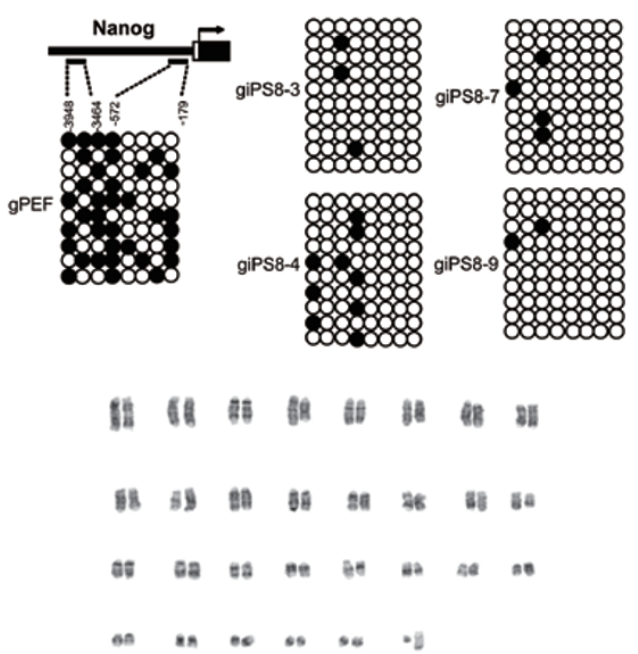

I
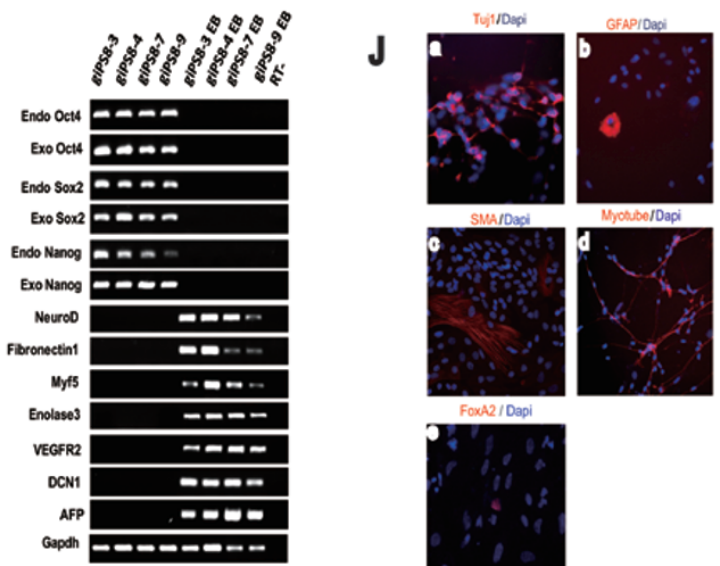

Mychibe/Dapi
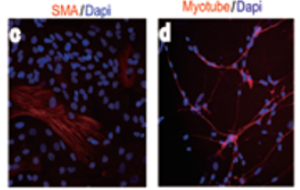

FoxA2/Dapi
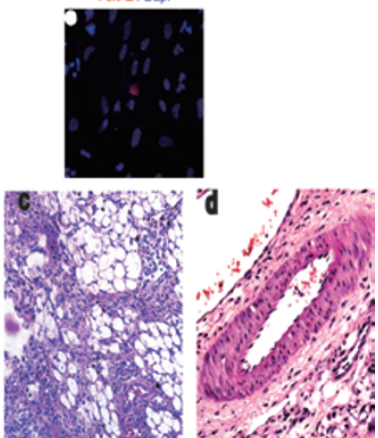
giPSCs was approximately $24 \mathrm{~h}(25.46 \pm 0.6 \mathrm{~h}$ for giPS8$3,24.58 \pm 0.9 \mathrm{~h}$ for giPS8-4, $23.5 \pm 1.2 \mathrm{~h}$ for giPS8-7, and $23.5 \pm 1.1 \mathrm{~h}$ for giPS8-9), and the plating efficiency was approximately $58 \% \pm 9.4 \%$ for giPS $8-3,56 \% \pm$ $1.7 \%$ for giPS $8-4,53 \% \pm 1.7 \%$ for giPS $8-7$, and $66 \% \pm$ $3.6 \%$ for giPS8-9, respectively. giPSCs expressed ES cell markers including SSEA1, Tra-1-60, Tra-1-81, Rex1, and E-Cadherin (CDH1) (Figure 1C), but they do not express SSEA3 and SSEA4.

All of the four giPSC lines analyzed so far exhibit induction of endogenous Oct4, Sox2, and Nanog as calculated by quantitative PCR. Other undifferentiated ES cell markers, such as TDGF, Rex1, Dnmt3b, Dax1, and CDH1, were also expressed (Figure 1D). The expression of E-cadherin suggests that giPSCs are fully reprogrammed beyond the stable ground state of 'near pluripotency' (the state of so-called FAB-SCs) to the state of 'full pluripotency' (the state of ES cells) [9]. Absolute quantitative PCR was used to measure the mRNA copy number of Oct4, Nanog and Sox2 in giPSCs and human ES cells. We found that the expression levels of endogenous Oct4, Nanog and Sox 2 in giPSCs were comparable to those of hESCs (Figure 1E).

Bisulfite genomic sequencing analyses of the Nanog promoter showed that it was highly unmethylated in giPSC clones, whereas $\mathrm{CpG}$ dinucleotides in these regions were highly methylated in parental gPEFs (Figure 1F). These results indicate that the Nanog promoter was reactivated in the giPSCs. We also performed karyotyping analyses after passage 30, and our analyses suggested that the goat iPSC clones showed a normal karyotype of 58XY (Figure 1G).

The expression of exogenous genes was well maintained by DOX in the giPSCs (Figure 1H). When DOX was withdrawn, the exogenous genes were quickly down- regulated, and the giPSCs differentiated and no longer exhibited ES cell morphology (Figure 1H and data not shown), suggesting that certain growth factors or chemical inhibitors would be required in the culture medium in order to maintain the giPSCs in a pluripotent state.

EBs of the four goat iPS clones were observed after trypsinization and culture in DMEM $+10 \%$ FBS in $10-$ $\mathrm{cm}^{2}$ dishes. RT-PCR confirmed that the giPSCs could differentiate into all three germ layers in EBs, as evidenced by the expression of NeuroD (ectoderm), Fibronectin (ectoderm), Myf5 (mesoderm), Enolase3 (mesoderm), AFP (endoderm), and Defective in cullin neddylation 1 (DCN1, endoderm). In contrast, endogenous Oct3/4, Sox2, and Nanog expression was markedly decreased (Figure 1I). After 8 days in suspension culture, we transferred these EB-like structures to gelatin-coated plates and continued cultivation for another 8 days. Attached cells showed various types of morphologies. Immunocytochemistry detected cells positive for Tuj1 (bIIItubulin, ectoderm), glial fibrillary acidic protein (GFAP, ectoderm), $\alpha$-smooth muscle actin ( $\alpha$-SMA, mesoderm), Myotube (mesoderm), and FoxA2 (endoderm) (Figure $1 \mathrm{~J})$. These data demonstrate that these giPSCs can differentiate into three germ layers in vitro.

In order to determine the differentiation capacity of giPSCs in vivo, we injected giPSCs intramuscularly into non-obese diabetic/severe combined immune deficient (NOD/SCID) mice. Four weeks after injection, tumor formation was observed. Histological examination showed that the tumor contained various tissues of three germ layers, including keratinocyte cells (ectoderm), smooth muscle (mesoderm), adipose tissue (mesoderm) and gut-like epithelial tissues (endoderm) (Figure 1K).

In conclusion, goat iPS cells have been successfully generated, which may help reveal the properties of goat

Figure 1 (A) Time line of the reprogramming protocol applied. (B) A comparison of the number of ESC-like colonies and APpositive colonies generated from PEFs in different conditions; $n=3$. Error bars indicate standard deviations. Abbreviations: 8F, 8 factors; T, SV40, the large T antigen; t, hTert; O, Oct4; S, Sox2; M, cMyc; K, Klf4; L, Lin28; N, Nanog. (C) GiPSCs exhibit a typical ES-cell like morphology. (a) Morphology of partially reprogrammed goat ipSCs; (b) morphology of giPSCs; (c) typical giPSC colony; (d) high magnification of the giPSCs; and staining patterns for AP (e), SSEA1 (f), SSEA3 (g), SSEA4 (h), REX1 (i), Tra-1-60 (j), Tra-1-81 (k) and E-cadherin (I). (D) Reverse transcription-polymerase chain reaction (RT-PCR) analysis of the giPSC lines compared with the gene expression observed in goat primary cells. Abbreviation: -RT, minus reverse transcriptase. (E) Quantitative reverse transcription-PCR analyses of endogenous Oct4, Nanog and Sox2 expression in hESCs, giPSCs, and parental somatic cell populations. The y-axis indicates mRNA copy number of the gene of interest per $10^{6}$ copies of GAPDH. (F) Bisulfite genomic sequencing of the promoter regions of Nanog. The open and closed circles indicate unmethylated and methylated CpGs, respectively. (G) The giPSCs at passage 30 showed a normal 58XY karyotype. (H) Exogenous gene down-regulation in giPSCs after DOX withdrawal. (I) Real-time RT-PCR analysis of relative transcript concentrations of pluripotency and lineage-specific genes in giPSCs and giPSC-derived EBs. (J) Immunocytochemistry of Tuj1 (a), GFAP (b), a-smooth muscle actin (c), Myotube (d), and FoxA2 (e). Nuclei were stained with Hoechst 33342 (blue). (K) Hematoxylin-eosin staining of teratomas derived from giPSCs. Teratomas are composed of various tissue types: (a) keratinized epithelium (ectoderm), (b) smooth muscle (mesoderm), (c) adipose tissue (mesoderm), and (d) gut-like epithelium (endoderm). 
ES cells. GiPSCs showed a morphology similar to mouse ESCs and expressed SSEA1, Tra-1-60 and Tra-1-81, but were negative for SSEA3 and SSEA4. The generation of goat iPS cells provides information for the establishment of and identification criteria for goat ES cells in the future. It is likely that goat ES cells may share surface marker expression patterns with giPSCs.

Moreover, giPSCs can form EBs and teratomas, which proves their pluripotency potential in vitro and in vivo. In addition, giPSCs are able to proliferate for a long period of time without differentiation. These properties provide an opportunity for using the homologous recombination technique in giPSCs. The successful demonstration of germline transmission[10] and homologous recombination in mouse iPS cells[4] encourages us to propose that giPSCs might also be directly useful for the generation of knock-out and knock-in goats either through the chimera technology that is well established for producing genetically modified mice, or through improving the efficiency of the somatic cell nuclear transfer technology, since the efficiency of site-specific recombination technology is higher for ES cells or pluripotent stem cells than for somatic cells.

We speculate that it is necessary to shut off the exogenous gene expression for three-germ-layer differentiation, since iPSCs with constitutive exogenous gene expression failed to differentiate into three germ layers in teratomas, while iPSCs generated by the tet-on system allowed for teratoma differentiation when DOX was withdrawn. We propose that the SV40 large T antigen is very important for the generation of giPSCs. We found that colony-like cells could not even be generated without the SV40 large T antigen. We found that it was critical to trypsinize and passage transduced goat fibroblasts during the reprogramming progress. Adding SV40 large $\mathrm{T}$ antigen and hTERT obviously increased the proliferation of cells. By trypsinizing and passaging cells once, we obtained AP-positive colonies at about 26 days after viral transduction. Without trypsinizing and passaging, no ESC-like colonies could be obtained. All of the primer sequences for PCR, RT-PCR and Bisulfite genomic sequencing were supplied in Supplementary information, Table S1.

\section{Acknowledgments}

We thank the Third World Academy of Sciences and the Chinese Academy of Sciences for technical assistance, funds and fellowship. We thank Huimin Dai, Weiying Fang and Qing Tian for technical assistance. This research was partially supported by grants from the National Key Basic Research and Development Program of China (2007CB947902, 2007CB948003, 2007 CB 947100 , 2009 CB 941101,2009 C B 940903 ,
2011CB965101), the National Natural Science Foundation of China (30971682, 30901019, 31025016), the Ministry of Agriculture (2009ZX08010-016B), the National High Technology Research and Development Program (863 program) of China (2010AA100504), and the Chinese Academy of Sciences Knowledge Creation Program (KSCX2-YW-R-223).

Jiangtao Ren ${ }^{1, *}$, Yongjun Pak $^{2,{ }^{*}}$, Lixiazi $\mathrm{He}^{1}$, Lei Qian ${ }^{1}$, Yijun $\mathrm{Gu}^{1}$, Hui $\mathrm{Li}^{3}$, Lingjun Rao ${ }^{1}$, Jing Liao, Chun Cui ${ }^{1,4}$, Xun $\mathrm{Xu}^{5}$, Jinqiu Zhou ${ }^{6}$, Hakchol $\mathrm{Ri}^{2}$, Lei Xiao ${ }^{1,4}$

${ }^{1}$ Laboratory of Molecular Cell Biology, Institute of Biochemistry and Cell Biology, Cell Bank, Stem Cell Bank, Shanghai Institutes for Biological Sciences, Chinese Academy of Sciences, Shanghai 200031, China; ${ }^{2}$ Cloning Center, Biology Branch, Academy of Sciences, Korea DPR; ${ }^{3}$ Xiangtan Central Hospital, Xiangtan 411100, China; ${ }^{4}$ College of Animal Sciences, Zhejiang University, Hangzhou 310058, China; ${ }^{5}$ Department of Animal Toxinology, Kunming Institute of Zoology, Chinese Academy of Science, Kunming 650000, China; ${ }^{6}$ State Key Laboratory of Molecular Biology, Institute of Biochemistry and Cell Biology, Shanghai Institutes for Biological Sciences, Chinese Academy of Sciences, Shanghai 200031, China

*These two authors contributed equally to this work.

Correspondence: Lei Xiao ${ }^{\mathrm{a}}$, Hakchol Ri ${ }^{\mathrm{b}}$

${ }^{\mathrm{a} T e l}$ : +86-21-54921386; Fax: +86-21-54921388

E-mail: leixiao@sibs.ac.cn

${ }^{\mathrm{b}}$ Tel:+86-21-54921365; Fax: +86-21-54921387

E-mail: sibslixuezhe@126.com

\section{References}

1 Ohkoshi K, Takahashi S, Koyama S, et al. In vitro oocyte culture and somatic cell nuclear transfer used to produce a liveborn cloned goat. Cloning Stem Cells 2003; 5:109-115.

2 Thomas KR, Capecchi MR. Site-directed mutagenesis by gene targeting in mouse embryo-derived stem cells. Cell 1987; 51:503-512.

3 Takahashi K, Yamanaka S. Induction of pluripotent stem cells from mouse embryonic and adult fibroblast cultures by defined factors. Cell 2006; 126:663-676.

4 Hanna J, Wernig M, Markoulaki S, et al. Treatment of sickle cell anemia mouse model with iPS cells generated from autologous skin. Science 2007; 318:1920-1923.

5 Liao J, Cui C, Chen S, et al. Generation of induced pluripotent stem cell lines from adult rat cells. Cell Stem Cell 2009; 4:1115.

$6 \mathrm{Wu}$ Z, Chen J, Ren J, et al. Generation of pig induced pluripotent stem cells with a drug-inducible system. J Mol Cell Biol 2009; 1:46-54.

7 Liao J, Wu Z, Wang Y, et al. Enhanced efficiency of generating induced pluripotent stem (iPS) cells from human somatic cells by a combination of six transcription factors. Cell Res 2008; 18:600-603.

8 Mali $\mathrm{P}$, Ye Z, Hommond $\mathrm{HH}$, et al. Improved efficiency and 
pace of generating induced pluripotent stem cells from human adult and fetal fibroblasts. Stem Cells 2008; 26:1998-2005.

9 Chou YF, Chen HH, Eijpe M, et al. The growth factor environment defines distinct pluripotent ground states in novel blastocyst-derived stem cells. Cell 2008; 135:449-461.

10 Okita K, Ichisaka T, Yamanaka S. Generation of germlinecompetent induced pluripotent stem cells. Nature 2007; 448:313-317.

(Supplementary information is linked to the online version of the paper on the Cell Research website.) 